\title{
ORIGINAL
}

\section{CORRESPONDENCIAS ENTRE CIE-10 Y CIE-9 PARA LAS LISTAS DE CAUSAS DE MUERTE DEL INSTITUTO NACIONAL DE ESTADÍSTICA Y DE LA REGIÓN DE MURCIA}

\section{Lluís Cirera Suárez (1), Miguel Rodríguez Barranco (1), Emilia Madrigal de Torres (1), Jesús Carrillo Prieto (2), Augusto Hasiak Santo (3), Roberto Augusto Becker (4), Aurelio Tobías Garcés (5), y Carmen Navarro Sánchez (1) del grupo COMPARA}

(1) Servicio de Epidemiología. Dirección General de Salud Pública. Consejería de Sanidad. Murcia. España.

(2) Área de Estadísticas Sanitarias, Instituto Nacional de Estadística. Madrid. España.

(3) Departamento de Epidemiología, Facultad de Salud Pública, Universidad de San Pablo, San Pablo, Brasil.

(4) Programa de Análisis de Salud y Sistemas de Información Sanitaria, Organización Panamericana de Salud. Washington. Estados Unidos.

(5) Departamento de Matemáticas. Universidad Autónoma de Barcelona. Bellaterra. España.

\section{RESUMEN}

Fundamento: Diversos países han realizado estudios de comparabilidad entre las revisiones $10^{\mathrm{a}}$ y $9^{\mathrm{a}}$ de la Clasificación Internacional de Enfermedades para listas agregadas de causas de muerte. En España el proyecto COMPARA pretende evaluar el impacto del cambio de revisión.

Métodos: Estudio epidemiológico transversal descriptivo de 88.048 defunciones inscritas en 1999 en España con la causa de muerte doblemente codificada en CIE-9 y 10. Se establecen las correspondencias teóricas entre CIE en las listas INE y Murcia. Se calculan las razones de comparabilidad e intervalos de confianza y el índice kappa global.

Resultados: Descienden las enfermedades infecciosas $(-1,7 \%)$, las hepatitis víricas $(-12,3 \%)$, mientras que aumenta el Sida $(5,7 \%)$. Las neoplasias aumentan el $0,3 \%$ por la incorporación del síndrome mielodisplásico $(55,2 \%)$. Se incrementa la diabetes mellitus $(2,1 \%)$ Se reducen los trastornos mentales al salir las demencias hacia la enfermedad de Alzheimer (28,6\%). Las enfermedades cardiovasculares descienden ligeramente $(-1,4 \%)$, sin impacto sobre las cerebrovasculares, aunque el infarto agudo de miocardio decrece $(-0,6 \%)$ con aumento de la enfermedad isquémica cardiaca $(0,3 \%)$. La neumonía decrece $(-12,5 \%)$ y la cirrosis hepática se aumenta $(4,3 \%)$. Las entidades mal definidas aumentan por la cesión de las insuficiencias cardiorrespiratorias. Las causas externas no varían sin que recojan la precisión de la CIE-9. La lista INE - 102 grupos obtiene un índice kappa del 95,4\%, similar a las variantes de Murcia.

Correspondencia:

Lluís Cirera.

Servicio de Epidemiología.

Consejería de Sanidad.

Ronda de Levante, 11.

30008 - Murcia,

Correo electrónico: Lluis.Cirera@carm.es
ABSTRACT

\section{Correspondences from 10th to 9th Revision of the International Classification of Diseases in the Causes of Death Lists of the National Institute of Statistics and the Regional Health Authority of Murcia in Spain}

Background: Different countries have conducted comparability studies between Revisions 10 and 9 of the International Classification of Diseases for aggregate lists of causes of death. In Spain, the COMPARA project was aimed at evaluating the impact of the revision change.

Methods: Descriptive cross-sectional epidemiological study of 88,048 deaths recorded in Spain in 1999 with the underlying cause of death doubled coded in ICD-9 and ICD-10. The theoretical correspondences between the ICD on the lists of the National Institute of Statistics and Murcia are established. The comparability rates and their confidence intervals, and the total kappa index were calculated.

Results: A decline in infectious diseases (-1.7\%) and viral hepatitis, $(-12.3 \%)$ declined under Tenth revision, while AIDS showed an increase $(5.7 \%)$. Neoplasms increased a little $(0.3 \%)$ with the inclusion of the Mielodisplasic Syndrome (55.2\%). Diabetes mellitus is increased $(2.1 \%)$. Mental disorders declined on dementia being shifted to Alzheimer's disease $(28.6 \%)$. Cardiovascular diseases dropped slightly $(-1.4 \%)$, without any impact on cerebrovascular diseases, although acute myocardial infarct decreased $(-0.6 \%)$ while ischemic heart disease increased $(0.3 \%)$. Pneumonia decreased $(-12.5 \%)$ and hepatic cirrhosis grows $(4.3 \%)$. Ill-defined conditions increased due to cardiorespiratory insufficiencies. The external causes show no change without including the accuracy of ICD-9. The National Institute of Statistics 102 groups list obtained a total kappa index of $95.4 \%$, similar to the Murcia variants. 
Conclusiones: Aunque el impacto global de la CIE-10 es menor, se deberían tener en cuenta las razones de comparabilidad significativas de los grupos de causas de muerte con diferencias absolutas importantes entre las revisiones.

Palabras clave: Clasificación Internacional de Enfermedades. CIE-10. CIE-9. Registros sanitarios. Estadísticas vitales. Causa de muerte. Razones de comparabilidad.
Conclusions: Although ICD-10 has a lesser overall impact, the significant comparability rates of the causes of death groups between the revisions with important absolute differences should be taken into account.

Key words: International Classification of Diseases. ICD-10. ICD-9. Medical Records. Vital Statistics. Cause of Death. Comparability Ratios. Bridge-coding. Spain.

\section{INTRODUCCIÓN}

España aplica la décima revisión de la Clasificación Estadística Internacional de Enfermedades (CIE-10) a las estadísticas de mortalidad desde el año $1999^{1}$.

La CIE-10 genera el cambio más importante desde la sexta revisión al aumentar el número de códigos, de categorías (grupos anatómicos o clínicos) y de capítulos (grandes grupos de causas o circunstancias del fallecimiento). Implanta la codificación alfanumérica. Varia, agrupa y elimina reglas de selección y modificación de la causa de muerte. Enumera las enfermedades originadas por el virus de inmunodeficiencia humana (VIH). Da una mayor y mejor información en neoplasias (tumores). A la vez que actualiza la definición de mortalidad perinatal, entre otros aspectos claves $^{2345}$.

El proyecto COMPARA ha publicado en España los resultados de las correspondencias entre CIE-10 y 9 por grandes causas de muerte $^{6}$, informando que el $3,6 \%$ de las defunciones cambia de grupo, debido al aumento $(36,4 \%)$ de las enfermedades infecciosas y parasitarias por la inclusión del SIDA. Esta misma causa y la pérdida del síndrome mielodisplásico producen la disminución de las enfermedades endocrinas, nutricionales y metabólicas $(-13,9 \%)$. Las neoplasias aumentan poco $(0,9 \%)$ e incorporan al síndrome mielodisplásico. Se reducen las enfermedades mentales y del comportamiento $(-7,5 \%)$ por la exclusión de la psicosis orgánica senil y presenil, que se clasifican en CIE-10 según la afectación orgánica, pasando al grupo de enfermedades del sistema nervioso. Las enfermedades del sistema circulatorio bajan ligeramente (-3\%) por la salida de la insuficiencia cardiorrespiratoria y la parada cardiaca hacia las entidades mal definidas, que incrementan su conjunto $(14,1 \%)$. Las enfermedades del sistema respiratorio aumentan $(4,8 \%)$ por la inclusión de la insuficiencia respiratoria, considerada una entidad mal definida en CIE-9. Las causas externas permanecen prácticamente invariables.

El INE elabora listas propias de tabulación de las causas de muerte en adecuación a nuestro patrón de mortalidad al igual que realizan otros organismos oficiales dentro y fuera de España ${ }^{8,9}$. Estas listas reducidas son distintas a las propuestas por la Organización Mundial de la Salud (OMS) ${ }^{10}$. A su vez el proyecto COMPARA permite estudiar el impacto del cambio de revisión en listas de causas más desagregadas y evaluar como estas causas modifican la comparabilidad de las grandes causas de muerte. Pretendemos establecer y cuantificar los cambios entre la décima y a la novena revisión en las agrupaciones de las causas de muerte de las listas del INE y las variantes de Murcia.

\section{MATERIAL Y MÉTODOS}

El proyecto COMPARA ${ }^{6}$ es un estudio epidemiológico transversal descriptivo de correspondencias entre revisiones de la CIE para las listas INE y Murcia, mediante la causa básica de 88.048 defunciones inscritas a lo largo del año 1999, en la ciudad Barcelona y en las CC AA de Andalucía (sólo el primer semestre), Cantabria, Murcia, Navarra (febrero a agosto) y País Vasco, doblemente codificadas en CIE-9 ${ }^{11}$ y CIE- $10^{12}$ por 
el personal habitual, siguiendo normas internacionales aplicadas en España. La asignación de los códigos ha sido manual partiendo del código en CIE-9 para después asignar el código en CIE-10. Los boletines estadísticos han sido revisados informaticamente para detectar errores e inconsistencias según causa, edad, sexo y otras variables ${ }^{13}$.

La categorización de la lista INE partió de la adaptación a la CIE-10 del manual de causas de defunción ${ }^{14,15}$, de la lista reducida INE en CIE-9, y de listas en CIE-10 de instituciones internacionales como la Short List de Eurostat o la Lista 6/67 de Organización Panamericana de Salud (OPS) ${ }^{16}$. El listado se elaboró en base a criterios de magnitud y relevancia. El INE, tras un periodo de información a los registros de mortalidad, lo dejó establecido en 102 epígrafes en junio de 2000, agrupando causas de 3 y 4 caracteres y grandes causas (cuadro 1). Las variantes de Murcia contienen rúbricas propias, como la enfermedad isquémica cardiaca ${ }^{17}$, las neoplasias malignas ${ }^{18}$,el cáncer de vesícula biliar, diferencia entre linfomas, agrupa a los cánceres de útero y a las enfermedades del hígado, e incluye cambios de criterio en los accidentes de tráfico en ciclistas (tabla 2) por agrupación en 86 y 66 causas (listas MU-86 y MU-66).

Se parte de una lista teórica de causas -las listas tabulares INE y Murcia-, se establecen las tablas de correspondencias entre CIE y se cruzan los códigos de cada epígrafe con la base de datos que contiene la doble codificación.

Para cuantificar las correspondencias de las listas entre las dos revisiones, se calcula el índice kappa como indicador de concordancia total ${ }^{19}$. Para estimar las correspondencias se aplica la metodología estadística internacional de comparabilidad, mediante las razones de comparabilidad de cada grupo de causas, estableciendo como el cociente entre el número defunciones asignadas a una misma entidad clasificada en CIE-10 y en CIE- $9{ }^{20}$ y sus respectivos intervalos de confianza al $95 \%{ }^{21}$. Es decir:

Intervalos de confianza por aproximación asindótica a la distribución normal:

$$
\begin{gathered}
\mathrm{EE}(\log \mathrm{r})=\sqrt{\operatorname{var}\left(\log d_{i, C I E-10}\right)+\operatorname{var}\left(\log d_{i, C I E-9}\right)-2 \operatorname{cov}\left(\log d_{i, C I E-10}, \log d_{i, C I E-9}\right)} \\
\text { Donde: } \operatorname{var}\left(\log d_{i, C I E-10}\right)=\frac{\operatorname{var} d_{i, C I E-10}}{\left(d_{i, C I E-10}\right)^{2}} ; \operatorname{var}\left(\log d_{i, C I E-9}\right)=\frac{\operatorname{var} d_{i, C I E-9}}{\left(d_{i, C I E-9}\right)^{2}} ; \\
\operatorname{cov}\left(\log d_{i, C I E-10}, \log d_{i, C I E-9}\right)=\frac{\operatorname{cov}\left(d_{i, C I E-10}, d_{i, C I E-9}\right)}{d_{i, C I E-10} d_{i, C I E-9}} ;
\end{gathered}
$$$$
\operatorname{var}\left(d_{i, C I E-10}\right)=\mathrm{n}\left(\mathrm{p}_{3}\left(1-\mathrm{p}_{3}\right)\right) ; \operatorname{Var}\left(d_{i, C I E-9}\right)=\mathrm{n}\left(\mathrm{p}_{2}\left(1-\mathrm{p}_{2}\right)\right) ; \operatorname{Cov}\left(d_{i, C I E-10} d_{i, C I E-9}\right)=-\mathrm{n}\left(\mathrm{p}_{3} \mathrm{p}_{2}\right) ;
$$

Intervalo de confianza al $95 \%: \mathrm{r}$. $\exp ( \pm 1,96 \mathrm{EE}(\log (\mathrm{r}))$ para una distribución normal a dos colas.

i = entidad nosológica de la lista.

$\mathrm{n}=$ número total de defunciones codificadas en CIE-9 y CIE-10. $\mathrm{p}_{1}=$ proporción de defunciones concordantes en CIE-9 y CIE-10.

$\mathrm{p}_{2}=$ proporción de defunciones codificadas en CIE-10 y no en CIE-9.

$\mathrm{p}_{3}=$ proporción de defunciones codificadas en CIE-9 y no en CIE-10. 
Tabla 1

Correspondencias de códigos CIE-10 a 9 de la lista INE de 102 grupos causas de muerte

\begin{tabular}{|c|c|c|c|}
\hline & Lista INE & códigos CIE-10 & códigos CIE-9 \\
\hline \multirow[t]{9}{*}{ I. $1-8$} & Enfermedades infecciosas y parasitarias $^{1}$ & $\mathrm{~A} 00-\mathrm{B} 99, \mathrm{R} 75$ & $001-139,279.5 .6,795.8$ \\
\hline & 1Enfermedades infecciosas intestinales & $\mathrm{A} 00-\mathrm{A} 09$ & $001-009$ \\
\hline & 2Tuberculosis y sus efectos tardíos & A15-A19, B90 & $010-018,137$ \\
\hline & 3Infecciones meningocócicas & A39 & 36 \\
\hline & 4Septicemia & $\mathrm{A} 40, \mathrm{~A} 41$ & 38 \\
\hline & 5Hepatitis víricas & B15-B19 & 70 \\
\hline & 6SIDA & B20-B24 & 279.5 .6 \\
\hline & $\begin{array}{l}7 \mathrm{VIH}+(\text { portador, evidencias de laboratorio del } \\
\mathrm{VIH}, \ldots)^{1}\end{array}$ & R75 & 795.8 \\
\hline & $\begin{array}{l}\text { 8Resto de enfermedades infecciosas y parasitarias y } \\
\text { sus efectos tardíos }\end{array}$ & Resto A00-B99 & Resto 001-139 \\
\hline \multirow[t]{34}{*}{ II. 9-41 } & Tumores / Neoplasias & C00-D48 & $140-239,273.1 .3,289.8$ \\
\hline & 9Cáncer del labio, de la cavidad bucal y de la faringe & $\mathrm{C} 00-\mathrm{C} 14$ & $140-149$ \\
\hline & 10Cáncer del esófago & $\mathrm{C} 15$ & 150 \\
\hline & 11Cáncer del estómago & $\mathrm{C} 16$ & 151 \\
\hline & 12Cáncer del colon & $\mathrm{C} 18$ & 153 \\
\hline & $\begin{array}{l}\text { 13 Cáncer del recto, de la porción rectosigmoide y del } \\
\text { ano }\end{array}$ & $\mathrm{C} 19-\mathrm{C} 21$ & 154 \\
\hline & 14Cáncer del hígado y vías biliares intrahepáticas & $\mathrm{C} 22$ & 155 \\
\hline & 15Cáncer del páncreas & $\mathrm{C} 25$ & 157 \\
\hline & 16Otros cánceres digestivos & Resto C15-C26, C45.1, C48 & Resto $150-159$ \\
\hline & 17Cáncer de la laringe & $\mathrm{C} 32$ & 161 \\
\hline & 18Cáncer de la tráquea, de los bronquios y del pulmón & C C33, C34 & 162 \\
\hline & 19Otros cánceres respiratorios e intratorácicos & Resto C30-C39, C45.0.2 & Resto $160-165$ \\
\hline & 20Cánceres del hueso y de los cartílagos articulares & $\mathrm{C} 40, \mathrm{C} 41$ & 170 \\
\hline & 21 Melanoma maligno de la piel & $\mathrm{C} 43$ & 172 \\
\hline & 22Otros cánceres de la piel y de los tejidos blandos & $\begin{array}{l}\text { C44-C47, C49 (excepto } \\
\text { C45.0.1.2) }\end{array}$ & 171,173 \\
\hline & 23Cáncer de la mama & $\mathrm{C} 50$ & 174,175 \\
\hline & 24Cáncer del cuello del útero & $\mathrm{C} 53$ & 180 \\
\hline & 25Cáncer de otras partes del útero & $\mathrm{C} 54, \mathrm{C} 55$ & 179,182 \\
\hline & 26Cáncer del ovario & $\mathrm{C} 56$ & 183.0 \\
\hline & 27Cánceres de otros órganos genitales femeninos & Resto C51-C58 & Resto $179-184$ \\
\hline & 28Cáncer de la próstata & C61 & 185 \\
\hline & 29Cánceres de otros órganos genitales masculinos & Resto C60-C63 & 186,187 \\
\hline & 30Cáncer del riñón, excepto pelvis renal & C64 & 189.0 \\
\hline & 31 Cáncer de la vejiga & C67 & 188 \\
\hline & 32Otros cánceres de las vías urinarias & Resto C64-C68 & Resto $188-189$ \\
\hline & 33Cáncer del encéfalo & C71 & 191 \\
\hline & 34Otros cánceres neurológicos y endocrinos & Resto C69-C75 & Resto $190-194$ \\
\hline & $\begin{array}{l}\text { 35Cáncer de sitios mal definidos, secundarios y de } \\
\text { sitios no especificados }\end{array}$ & C76-C80, C97 & $195-199$ \\
\hline & $\begin{array}{l}\text { 36Cánceres del tejido linfático, de los órganos } \\
\text { hematopoyéticos y de tejidos afines }\end{array}$ & C81-C90, C96 & $200-203,273.3$ \\
\hline & 37Leucemia & C91-C95 & $204-208$ \\
\hline & 38Tumores in situ & D00-D09 & $230-234$ \\
\hline & 39Tumores benignos & D10-D36 & $210-229$ \\
\hline & 40 Síndrome mielodisplásico ${ }^{2}$ & D46 & 289.8 \\
\hline & $\begin{array}{l}41 \text { Otros tumores de comportamiento incierto o } \\
\text { desconocido }\end{array}$ & D37-D45, D47, D48 & $235-239,273.1$ \\
\hline \multirow[t]{3}{*}{ III. 42-43 } & $\begin{array}{l}\text { Enfermedades de la sangre y de los órganos } \\
\text { hematopoyéticos, y ciertos trastornos que } \\
\text { afectan al mecanismo de la inmunidad }\end{array}$ & D50-D89 & $\begin{array}{l}273.0 .2,279-289 \text { (excepto } \\
279.5 .6,289.8)\end{array}$ \\
\hline & $\begin{array}{l}\text { 42Enfermedades de la sangre y de los órganos } \\
\text { hematopoyéticos }\end{array}$ & D50-D76 & $280-289$ (excepto 289.8 ) \\
\hline & $\begin{array}{l}\text { 43 Ciertos trastornos que afectan al mecanismo de la } \\
\text { inmunidad }\end{array}$ & D80-D89 & $\begin{array}{l}273.0 .2,279 \text { (excepto } \\
279.5 .6)\end{array}$ \\
\hline
\end{tabular}


Tabla 1 (Cont.)

Correspondencias de códigos CIE-10 a 9 de la lista INE de 102 grupos causas de muerte

\begin{tabular}{|c|c|c|c|}
\hline & Lista INE & códigos CIE-10 & códigos CIE-9 \\
\hline \multirow[t]{3}{*}{ IV. 44-45 } & $\begin{array}{l}\text { Enfermedades endocrinas, nutricionales y } \\
\text { metabólicas }\end{array}$ & E00-E90 & $\begin{array}{l}240-278,330.0 .1 \text { (excepto } \\
273.0 .1 .2 .3,274)\end{array}$ \\
\hline & 44Diabetes mellitus & E10-E14 & 250 \\
\hline & $\begin{array}{l}45 \text { Otras enfermedades endocrinas, nutricionales y } \\
\text { metabólicas }\end{array}$ & Resto E00-E90 & $\begin{array}{l}\text { Resto 240-278, 330.0.1 } \\
\text { (excepto 273.0.1.2.3, 274) }\end{array}$ \\
\hline \multirow[t]{5}{*}{ V. 46-49 } & Trastornos mentales y del comportamiento & F00-F99 & $290-319$ \\
\hline & 46 Trastornos mentales orgánicos, senil y presenil & F00-F09 & 290 \\
\hline & 47Trastornos mentales debidos al uso de alcohol & $\mathrm{F} 10$ & 291,303 \\
\hline & $\begin{array}{l}\text { 48 Trastornos mentales debidos al uso de drogas } \\
\text { (drogodependencias, toxicomanía) }\end{array}$ & F11-F16, F18, F19 & 304,305 \\
\hline & 49Otros trastornos mentales y del comportamiento & Resto F00-F99 & Resto 290-319 \\
\hline \multirow[t]{4}{*}{ VI-VIII. 50-52 } & $\begin{array}{l}\text { Enfermedades del sistema nervioso y de los } \\
\text { órganos de los sentidos }\end{array}$ & G00-H95 & $\begin{array}{l}320-389,435 \text { (excepto } \\
330.0 .1)\end{array}$ \\
\hline & 50Meningitis (otras en 3 ) & G00-G03 & $320-322$ \\
\hline & 51Enfermedad de Alzheimer & G30 & 331.0 \\
\hline & $\begin{array}{l}\text { 52Otras enfermedades del sistema nervioso y de los } \\
\text { órganos de los sentidos }\end{array}$ & Resto de G00-H95 & $\begin{array}{l}\text { Resto 320-389, } 435 \text { (excepto } \\
330.0 .1 \text { ) }\end{array}$ \\
\hline \multirow[t]{10}{*}{ IX. 53-61 } & Enfermedades del sistema circulatorio ${ }^{3}$ & I00-I99 & $\begin{array}{l}390-459,(\text { excepto } 427.5 \\
435,446,459.0)\end{array}$ \\
\hline & 53Enfermedades cardíacas reumáticas crónicas & I05-I09 & $393-398$ \\
\hline & 54Enfermedades hipertensivas & $\mathrm{I} 10-\mathrm{I} 15$ & $401-405$ \\
\hline & 55Infarto agudo de miocardio & $\mathrm{I} 21$ & 410 \\
\hline & 56Otras enfermedades isquémicas del corazón & $\mathrm{I} 20, \mathrm{I} 22-\mathrm{I} 25$ & $411-414$ \\
\hline & 57Insuficiencia cardiaca & $\mathrm{I} 50$ & 428 \\
\hline & 58 Otras enfermedades del corazón & I00-I02, I26-I49, I51, I52 & $\begin{array}{l}390-392,415-417,420-427 \\
429 \text { (excepto } 427.5 \text { ) }\end{array}$ \\
\hline & 59Enfermedades cerebrovasculares & I60-I69 & $430-434,436-438$ \\
\hline & 60Aterosclerosis & $\mathrm{I} 70$ & 440 \\
\hline & 61 Otras enfermedades de los vasos sanguíneos & I71-I99 & 441-459 (excepto 446, 459.0) \\
\hline \multirow[t]{7}{*}{ X. 62-67 } & Enfermedades del sistema respiratorio & J00-J99 & $460-519,786.0$ \\
\hline & 62 Influenza (gripe) & $\mathrm{J} 10, \mathrm{~J} 11$ & 487 \\
\hline & 63Neumonía & J12-J18 & $480-486$ \\
\hline & $\begin{array}{l}\text { 64Enfermedades crónicas de las vías respiratorias } \\
\text { inferiores (excepto asma) }\end{array}$ & J40-J44, J47 & $490-492,494-496$ \\
\hline & 65 Asma & $\mathrm{J} 45, \mathrm{~J} 46$ & 493 \\
\hline & 66Insuficiencia respiratoria 4 & J96 & 786.0 \\
\hline & 67Otras enfermedades del sistema respiratorio & Resto J00-J99 & Resto 460-519 \\
\hline \multirow[t]{6}{*}{ XI. 68-72 } & Enfermedades del sistema digestivo & K00-K93 & $520-579$ \\
\hline & 68Úlcera de estómago, duodeno y yeyuno & $\mathrm{K} 25-\mathrm{K} 28$ & $531-534$ \\
\hline & 69Enteritis y colitis no infecciosas & K50-K52 & $555,556,558$ \\
\hline & 70Enfermedad vascular intestinal & K55 & 557 \\
\hline & 71 Cirrosis y otras enfermedades crónicas del hígado & $\begin{array}{l}\text { K70, K72.1, K73, K74, K76.1, } \\
\text { K76.9 }\end{array}$ & 571 \\
\hline & 72Otras enfermedades del sistema digestivo & Resto K00-K93 & Resto 520-579 \\
\hline XII. 73 & 73Enfermedades de la piel y del tejido subcutáneo & L00-L99 & $680-709$ \\
\hline \multirow[t]{4}{*}{ XIII. 74-76 } & $\begin{array}{l}\text { Enfermedades del sistema osteomuscular y del } \\
\text { tejido conjuntivo }\end{array}$ & M00-M99 & $274,446,710-739$ \\
\hline & 74Artritis reumatoide y osteoartrosis & M05, M06, M15-M19 & 714,715 \\
\hline & 75 Osteoporosis y fractura patológica & M80-M82, M84.4 & 733 \\
\hline & $\begin{array}{l}\text { 760tras enfermedades del sistema osteomuscular y de } \\
\text { tejido conjuntivo }\end{array}$ & Resto M00-M99 & Resto $710-739,274,446$ \\
\hline \multirow[t]{5}{*}{ XIV.77-80 } & Enfermedades del sistema genitourinario & N00-N99 & $580-629$ \\
\hline & 77Enfermedades del riñón y del uréter & $\mathrm{N} 00-\mathrm{N} 29$ & $580-594$ \\
\hline & 78Enfermedades de los órganos genitales masculinos & N40-N51 & $600-608$ \\
\hline & $\begin{array}{l}\text { 79Enfermedades de los órganos genitales femeninos } \\
\text { y trastornos de la mama }\end{array}$ & N60-N64, N70-N98 & $610,611,614-629$ \\
\hline & 80 Otras enfermedades del sistema genitourinario & Resto N00-N99 & Resto $580-629$ \\
\hline
\end{tabular}


Tabla 1 (Cont.)

Correspondencias de códigos CIE-10 a 9 de la lista INE de 102 grupos causas de muerte

\begin{tabular}{|c|c|c|c|}
\hline & Lista INE & códigos CIE-10 & códigos CIE-9 \\
\hline XV. 81 & 81 Embarazo, parto y puerperio & O00-099 & $630-676$ \\
\hline XVI. 82 & 82 Afecciones originadas en el periodo perinatal & P00-P96 & $760-779$ \\
\hline \multirow[t]{4}{*}{ XVII. 83-85 } & $\begin{array}{l}\text { Malformaciones congénitas, deformidades y } \\
\text { anomalías cromosómicas }\end{array}$ & Q00-Q99 & $740-759$ \\
\hline & 83 Malformaciones congénitas del sistema nervioso & Q00-Q07 & $740-742$ \\
\hline & 84Malformaciones congénitas del sistema circulatorio & Q20-Q28 & $745-747$ \\
\hline & $\begin{array}{l}\text { 85 Otras malformaciones congénitas, deformidades y } \\
\text { anomalías cromosómicas }\end{array}$ & Resto Q00-Q99 & Resto 740-759 \\
\hline \multirow[t]{5}{*}{ XVIII. 86-89 } & $\begin{array}{l}\text { Síntomas, signos y hallazgos anormales clínicos y } \\
\text { de laboratorio, NCOP }\end{array}$ & R00-R74, R76-R99 & $\begin{array}{l}427.5,459.0,780- \\
799(\text { excepto } 786.0,795.8)\end{array}$ \\
\hline & $\begin{array}{l}\text { 86Paro cardíaco, muerte sin asistencia y otra causa } \\
\text { desconocida }{ }^{3}\end{array}$ & R98, R99 & $427.5,798.9,799.9$ \\
\hline & 87 Senilidad & R54 & 797 \\
\hline & 88Muerte súbita infantil & R95 & 798.0 \\
\hline & $\begin{array}{l}89 \text { Resto de síntomas, signos y hallazgos anormales, } \\
\text { clínicos y de laboratorio, NCOP }\end{array}$ & Resto R00-R74, R76-R99 & $\begin{array}{l}459.0, \text { Resto } 780-799 \\
\text { (excepto } 786.0,795.8 \text { ) }\end{array}$ \\
\hline \multirow[t]{14}{*}{ XX. 90-102 } & Causas externas & V01-Y89 & E800-E999 \\
\hline & 90 Accidentes de tráfico de vehículos a motor & $\begin{array}{l}\text { V02-V04 .1.9 V09.2.3V } 12-V 14 \\
3.4 .5 .9 \text { V19.4.5.6.9 V20-V28 } \\
\text { 3.4.5.9 V29.4.5.6.9 V30- } \\
\text { V38.4.5.6.7.9 V39.4.5.6.9 V40 - } \\
\text { V48.4.5.6.7.9 V49.4.5.6.9 V50 - } \\
\text { V58.4.5.6.7.9 V59.4.5.6.9 V60 - } \\
\text { V68.4.5.6.7.9 V69.4.5.6.9 V70 - } \\
\text { V78.4.5.6.7.9 V79.4.5.6.9 } \\
\text { V80.3.4.5 V81.1 V82.1 } \\
\text { V83.0.1.2.3 V84.0.1.2.3 } \\
\text { V85.0.1.2.3 V86.0.1.2.3 } \\
\text { V87.0.1.2.3.4.5.6.7.8 V89.2.9 }\end{array}$ & $\mathrm{E} 810-\mathrm{E} 819$ \\
\hline & 91 Otros accidentes de transporte & Resto de V01-V99 & E800-E807, E820-E848 \\
\hline & 92 Caídas accidentales & W00-W19 & E880-E888 (excepto E887) \\
\hline & 93 Ahogamiento, sumersión y sofocación accidentales & W65-W84 & E910-E915 \\
\hline & 94 Accidentes por fuego, humo y sustancias calientes & $\mathrm{X} 00-\mathrm{X} 19$ & E890-E899, E924 \\
\hline & $\begin{array}{l}\text { 95 Envenenamiento accidental por psicofármacos y } \\
\text { drogas de abuso }\end{array}$ & $\mathrm{X} 41, \mathrm{X} 42, \mathrm{X} 44, \mathrm{X} 45$ & $\begin{array}{l}\mathrm{E} 850.0 .8 .9, \mathrm{E} 851-\mathrm{E} 855 \\
\mathrm{E} 858.9, \mathrm{E} 860\end{array}$ \\
\hline & 960tros envenenamientos accidentales & Resto de X40-X49 & Resto de E850-E869 \\
\hline & 97Otros accidentes & Resto W00-X59 & $\begin{array}{l}\text { Resto E800-E848, Resto } \\
\text { E880-E928 }\end{array}$ \\
\hline & 98 Suicidio y lesiones autoinfligidas & X60-X84 & E950-E959 \\
\hline & 99 Agresiones (homicidio) & $\mathrm{X} 85-\mathrm{Y} 09$ & E960-E969 \\
\hline & 100Eventos de intención no determinada & Y10-Y34 & E980-E989 \\
\hline & 101 Complicaciones de la atención médica y quirúrgica & Y40-Y84 & E870-E879, E930-E949 \\
\hline & 102 Otras causas externas y sus efectos tardíos & Resto Y35-Y89 & Resto E929-E999 \\
\hline
\end{tabular}

CIE = Clasificación Internacional de Enfermedades

1 Por motivos de comparabilidad, se incluye VIH+ (R75) (795.8) en el capítulo I. Enfermedades infecciosas y parasitarias, aunque la CIE-10 lo encuadra en el capítulo XVIII Síntomas, signos y estados morbosos mal definidos.

2 Por motivos de comparabilidad, se desagrega el Síndrome mielodisplásico (D46) (289.8) ya que la CIE-9 lo encuadraba en el capítulo IV. Enfermedades de la sangre y de los órganos hematopoyéticos.

3 Por motivos de comparabilidad, se excluye el Paro cardíaco (R98,R99) (427.5) de las enf. del sist. circulatorio, ya que la CIE-10 lo encuadra en el capítulo XVIII. Síntomas, signos y estados morbosos mal definidos.

4 Por motivos de comparabilidad con la CIE-10, se desagrega la Insuficiencia respiratoria (J96) (786.0) ya que la CIE-9 lo encuadraba en el capítulo XVIII. Síntomas, signos y estados morbosos mal definidos.

$\mathrm{NCOP}=$ no codificadas en otra parte. 
Tabla 2

Correspondencias de códigos CIE-10 y 9 para las listas de causas muerte de Murcia con epígrafes distintos a la lista INE

\begin{tabular}{lcr}
\hline Epígrafes propios de las listas de Murcia & códigos CIE-10 & códigos CIE-9 \\
\hline Cáncer de vesícula biliar y de vías biliares extrahepáticas & C23-C24 & 156 \\
\hline Cáncer del útero & C53-C55 & $179-180,182$ \\
\hline Enfermedad de Hodgkin & C81 & 201 \\
\hline Linfomas no Hodgkin & C82-C85 & $200,202.0 .1 .2 .8$ \\
\hline Neoplasias malignas (cáncer) & C00-C97 & $140-208$ \\
\hline Enfermedades isquémicas del corazón & I20-I25 & $410-414$ \\
\hline Enfermedades del hígado & K70-K76 & $570-573$ \\
\hline
\end{tabular}

CIE = Clasificación Internacional de Enfermedades

La razón de comparabilidad se puede recalcular a partir de las tablas y expresarse en tantos decimales como sea necesario; y aplicarlas para mantener la continuidad de las series anuales, tanto a las defunciones como a las tasas:

$$
\begin{gathered}
\text { Tasa }{ }_{\text {CIE-10_ajustada-año }}= \\
r_{\text {CIE-10/CIE-9 }} \text { tasa }_{\text {CIE-9_ajustada-año }}
\end{gathered}
$$

Las tasas se expresan en las mismas unidades. Para la corrección a CIE-9 se calcula el cociente inverso.

El comentario de resultados se hace en función de la magnitud del cambio y de la significación estadística de las razones de comparabilidad.

\section{RESULTADOS}

En la tabla 3 se puede observar la matriz de correspondencias hasta el cuarto carácter en los grandes grupos de causas de muerte. En la tabla 4 se muestran los resultados de las correspondencias, las diferencias absolutas y las razones CIE-10 a CIE-9 para los grandes grupos y las 102 agrupaciones de causas de la lista INE. En la tabla 5 se incluyen los mismos indicadores de resultados para las categorías propias de las listas de Murcia.
El capítulo de las enfermedades infecciosas y parasitarias, refleja un descenso del $1,7 \%$, siendo las hepatitis víricas la causa especificada que más disminuye $(-12,3 \%)$. Sin embargo aumenta el SIDA (26 casos y $5,7 \%$ ), que proviene de las enfermedades de la inmunidad en CIE-9 (tabla 4).

El grupo de las neoplasias experimenta un incremento del $0,4 \%$ (88 casos) por el añadido de enfermedades de la sangre y de los órganos hematopoyéticos (-69 casos y -20,3\%) que van ahora al síndrome mielodisplásico (53 casos y 55,2\%). Las neoplasias malignas aumentan algo menos $(0,3 \%)$ (tabla 5$)$ por casi todas las rúbricas de otros cánceres de órganos bien localizados y de cánceres mal localizados o inespecificados o secundarios (14,2\%), que en CIE-9 se ubican en cáncer de pulmón, hueso-cartílago articular, labiocavidad bucal-faringe, próstata, colon, mama y en cáncer de vejiga (resultados no mostrados). También son más frecuentes en la CIE-10, los otros tumores malignos de la piel y de los tejidos blandos $(15,8 \%)$ por anexionarse a los tumores malignos mal definidos, inespecificados o secundarios. El resto de las neoplasias no malignas disminuye un $3,7 \%$ por la exclusión de tumores malignos de la CIE-10 (tabla 5).

Las enfermedades endocrinas, nutricionales y metabólicas en CIE-10 aumentan (51 casos y 1,9\%) por la incorporación de defun- 


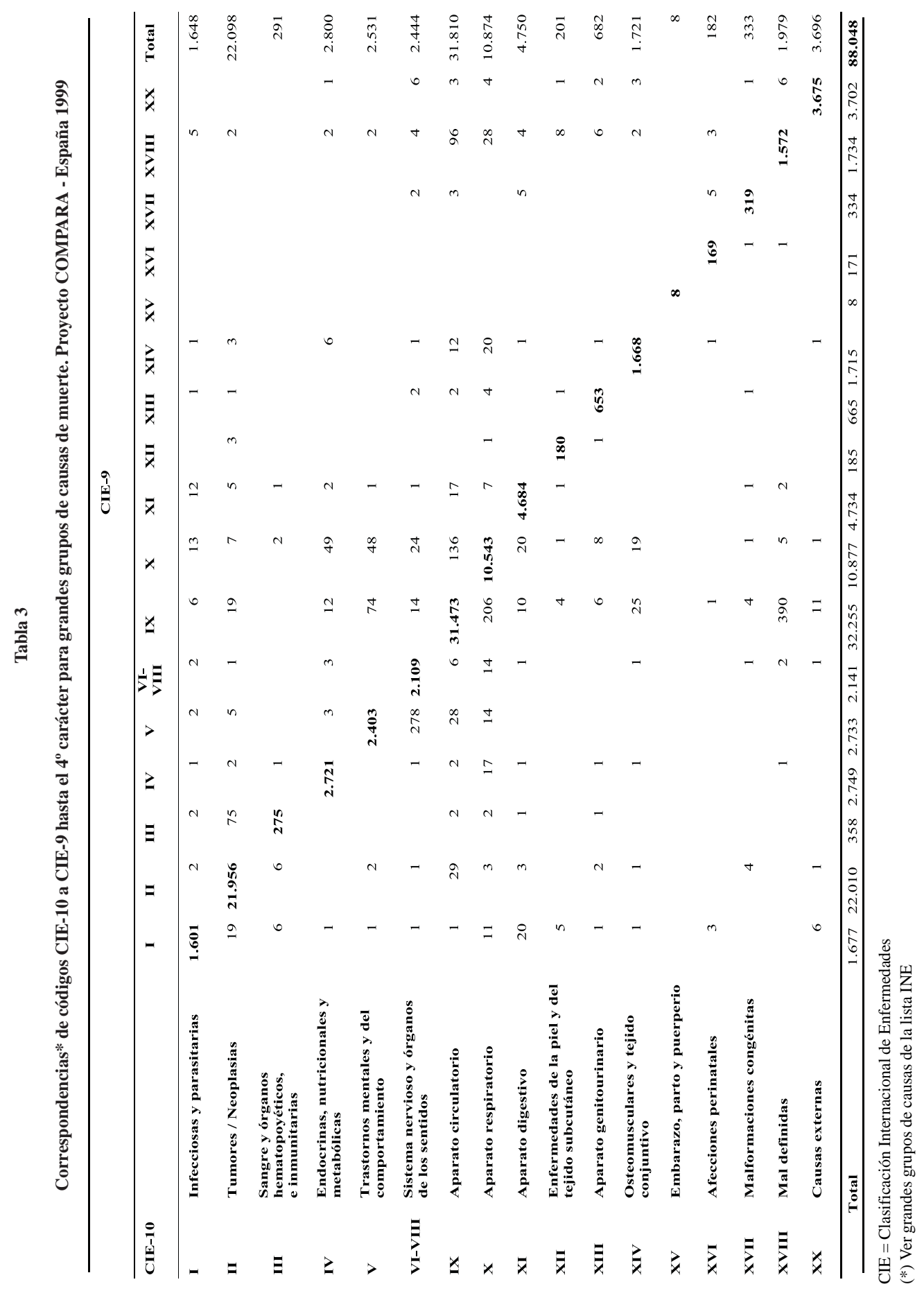


Tabla 4

Lista INE de 102 grupos de causas. Diferencias, razones de comparabilidad e intervalos de confianza al 95\% entre CIE-10 y CIE-9. Proyecto COMPARA - España 1999

\begin{tabular}{|c|c|c|c|c|c|c|c|}
\hline \multirow{2}{*}{$\begin{array}{l}\text { orden } \\
\text { I. }\end{array}$} & \multirow{2}{*}{$\begin{array}{c}\text { Lista INE } \\
\text { Enfermedades infecciosas y parasitarias }^{1}\end{array}$} & \multirow{2}{*}{$\begin{array}{c}\text { n } \\
\text { CIE-10 } \\
1.648\end{array}$} & \multirow{2}{*}{$\begin{array}{c}\mathbf{n} \\
\text { CIE-9 } \\
1.677\end{array}$} & \multirow{2}{*}{$\begin{array}{r}\text { Resta } \\
\text { CIE 10-9 } \\
-29\end{array}$} & \multirow{2}{*}{\begin{tabular}{c}
\multicolumn{1}{c}{ razón } \\
CIE 10/9 \\
0,983
\end{tabular}} & \multicolumn{2}{|c|}{$\begin{array}{l}\text { IC al } 95 \% \\
\text { Inferior Superior }\end{array}$} \\
\hline & & & & & & 0,970 & 0,996 \\
\hline & 1 Enfermedades infecciosas intestinales & 54 & 54 & 0 & 1,000 & 0,950 & 1,053 \\
\hline & 2 Tuberculosis y sus efectos tardíos & 211 & 219 & -8 & 0,963 & 0,934 & 0,994 \\
\hline & 3 Infecciones meningocócicas & 29 & 29 & 0 & 1,000 & 0,909 & 1,100 \\
\hline & 4 Septicemia & 529 & 539 & -10 & 0,981 & 0,958 & 1,005 \\
\hline & 5 Hepatitis víricas & 213 & 243 & -30 & 0,877 & 0,827 & 0,929 \\
\hline & 6 SIDA & 486 & 460 & 26 & 1,057 & 1,032 & 1,082 \\
\hline & $7 \mathrm{VIH}+(\text { portador, evidencias de laboratorio del } \mathrm{VIH}, \ldots)^{1}$ & 2 & 8 & -6 & 0,250 & 0,075 & 0,830 \\
\hline & $\begin{array}{l}8 \text { Resto de enfermedades infecciosas y parasitarias } \\
\text { y sus efectos tardíos }\end{array}$ & 124 & 125 & -1 & 0,992 & 0,888 & 1,108 \\
\hline II. & Tumores / Neoplasias & 22.098 & 22.010 & 88 & 1,004 & 1,003 & 1,005 \\
\hline & 9 Cáncer del labio, de la cavidad bucal y de la faringe & 568 & 589 & -21 & 0,964 & 0,943 & 0,986 \\
\hline & 0 Cáncer del esófago & 446 & 461 & -15 & 0,967 & 0,948 & 0,987 \\
\hline & 1 Cáncer del estómago & 1.257 & 1.271 & -14 & 0,989 & 0,982 & 0,996 \\
\hline & 2 Cáncer del colon & 2.006 & 2.015 & -9 & 0,996 & 0,989 & 1,002 \\
\hline & 3 Cáncer del recto, de la porción rectosigmoide y del ano & 608 & 606 & 2 & 1,003 & 0,988 & 1,019 \\
\hline & 4 Cáncer del hígado y vías biliares intrahepáticas & 1.036 & 1.038 & -2 & 0,998 & 0,986 & 1,010 \\
\hline & 5 Cáncer del páncreas & 827 & 834 & -7 & 0,992 & 0,981 & 1,002 \\
\hline & 6 Otros cánceres digestivos & 577 & 561 & 16 & 1,029 & 1,002 & 1,055 \\
\hline & 7 Cáncer de la laringe & 476 & 480 & -4 & 0,992 & 0,975 & 1,009 \\
\hline & 8 Cáncer de la tráquea, de los bronquios y del pulmón & 4.076 & 4.129 & -53 & 0,987 & 0,983 & 0,992 \\
\hline & 9 Otros cánceres respiratorios e intratorácicos & 134 & 131 & 3 & 1,023 & 0,953 & 1,098 \\
\hline & 0 Cánceres del hueso y de los cartílagos articulares & 63 & 89 & -26 & 0,708 & 0,605 & 0,828 \\
\hline & 1 Melanoma maligno de la piel & 172 & 186 & -14 & 0,925 & 0,876 & 0,976 \\
\hline & 2 Otros cánceres de la piel y de los tejidos blandos & 213 & 184 & 29 & 1,158 & 1,073 & 1,249 \\
\hline & 3 Cáncer de la mama & 1.431 & 1.455 & -24 & 0,984 & 0,974 & 0,993 \\
\hline & 4 Cáncer del cuello del útero & 123 & 124 & -1 & 0,992 & 0,965 & 1,020 \\
\hline & 5 Cáncer de otras partes del útero & 289 & 300 & -11 & 0,963 & 0,939 & 0,988 \\
\hline & 6 Cáncer del ovario & 356 & 357 & -1 & 0,997 & 0,985 & 1,010 \\
\hline & 7 Cánceres de otros órganos genitales femeninos & 126 & 120 & 6 & 1,050 & 0,998 & 1,104 \\
\hline & 8 Cáncer de la próstata & 1.240 & 1.249 & -9 & 0,993 & 0,983 & 1,003 \\
\hline & 9 Cánceres de otros órganos genitales masculinos & 45 & 44 & 1 & 1,023 & 0,979 & 1,069 \\
\hline & 0 Cáncer del riñón, excepto pelvis renal & 360 & 365 & -5 & 0,986 & 0,966 & 1,007 \\
\hline & 1 Cáncer de la vejiga & 916 & 922 & -6 & 0,993 & 0,980 & 1,008 \\
\hline & 2 Otros cánceres de las vías urinarias & 27 & 34 & -7 & 0,794 & 0,608 & 1,037 \\
\hline & 3 Cáncer del encéfalo & 499 & 495 & 4 & 1,008 & 0,992 & 1,024 \\
\hline & 4 Otros cánceres neurológicos y endocrinos & 129 & 130 & -1 & 0,992 & 0,948 & 1,038 \\
\hline & $\begin{array}{l}5 \text { Cáncer de sitios mal definidos, secundarios y } \\
\text { de sitios no especificados }\end{array}$ & 1.618 & 1.417 & 201 & 1,142 & 1,117 & 1,168 \\
\hline & $\begin{array}{l}6 \text { Cánceres del tejido linfático, de los órganos } \\
\text { hematopoyéticos y de tejidos afines }\end{array}$ & 1.033 & 1.030 & 3 & 1,003 & 0,991 & 1,015 \\
\hline & 7 Leucemia & 679 & 679 & 0 & 1,000 & 0,985 & 1,015 \\
\hline & 8 Tumores in situ & 2 & 0 & 2 & - & - & - \\
\hline & 9 Tumores benignos & 88 & 83 & 5 & 1,060 & 0,983 & 1,144 \\
\hline & 0 Síndrome mielodisplásico ${ }^{2}$ & 149 & 96 & 53 & 1,552 & 1,323 & 1,821 \\
\hline & 1 Otros tumores de comportamiento incierto o desconocido & 529 & 536 & -7 & 0,987 & 0,959 & 1,015 \\
\hline III. & $\begin{array}{l}\text { Enfermedades de la sangre y de los órganos } \\
\text { hematopoyéticos, y ciertos trastornos que afectan al } \\
\text { mecanismo de la inmunidad }\end{array}$ & 291 & 358 & -67 & 0,813 & 0,765 & 0,863 \\
\hline & $\begin{array}{l}2 \text { Enfermedades de la sangre y de los órganos } \\
\text { hematopoyéticos }\end{array}$ & 271 & 340 & -69 & 0,797 & 0,750 & 0,847 \\
\hline & $\begin{array}{l}3 \text { Ciertos trastornos que afectan al mecanismo de la } \\
\text { inmunidad }\end{array}$ & 20 & 18 & 2 & 1,111 & 0,801 & 1,540 \\
\hline
\end{tabular}


Tabla 4 (Cont.)

Lista INE de 102 grupos de causas. Diferencias, razones de comparabilidad e intervalos de confianza al $95 \%$ entre CIE-10 y CIE-9. Proyecto COMPARA - España 1999

\begin{tabular}{|c|c|c|c|c|c|c|c|}
\hline orden & Lista INE & \multirow{2}{*}{\begin{tabular}{c}
\multicolumn{1}{c}{$\mathbf{n}$} \\
CIE-10 \\
2.800
\end{tabular}} & \multirow{2}{*}{$\begin{array}{c}\mathbf{n} \\
\text { CIE-9 } \\
2.749\end{array}$} & \multirow{2}{*}{$\begin{array}{r}\begin{array}{c}\text { Resta } \\
\text { CIE 10-9 }\end{array} \\
51\end{array}$} & \multirow{2}{*}{$\begin{array}{r}\begin{array}{c}\text { razón } \\
\text { CIE 10/9 }\end{array} \\
1,019\end{array}$} & \multicolumn{2}{|c|}{$\begin{array}{c}\text { IC al 95\% } \\
\text { Inferior Superior }\end{array}$} \\
\hline$\overline{\mathrm{IV}}$. & $\begin{array}{l}\text { Enfermedades endocrinas, nutricionales y } \\
\text { metabólicas }\end{array}$ & & & & & 1,011 & 1,026 \\
\hline & 4 Diabetes mellitus & 2.389 & 2.341 & 48 & 1,021 & 1,013 & 1,028 \\
\hline & $\begin{array}{l}5 \text { Otras enfermedades endocrinas, nutricionales y } \\
\text { metabólicas }\end{array}$ & 411 & 408 & 3 & 1,007 & 0,984 & 1,032 \\
\hline V. & Trastornos mentales y del comportamiento & 2.531 & 2.733 & -202 & 0,926 & 0,911 & 0,941 \\
\hline & 6 Trastornos mentales orgánicos, senil y presenil & 2.393 & 2.524 & -131 & 0,948 & 0,932 & 0,965 \\
\hline & 7 Trastornos mentales debidos al uso de alcohol & 59 & 50 & 9 & 1,180 & 1,059 & 1,315 \\
\hline & $\begin{array}{l}8 \text { Trastornos mentales debidos al uso de drogas } \\
\text { (drogodependencias, toxicomanía) }\end{array}$ & 7 & 13 & -6 & 0,538 & 0,301 & 0,963 \\
\hline & 9 Otros trastornos mentales y del comportamiento & 72 & 146 & -74 & 0,493 & 0,413 & 0,589 \\
\hline VI-VIII. & $\begin{array}{l}\text { Enfermedades del sistema nervioso y de los órganos } \\
\text { de los sentidos }\end{array}$ & 2.444 & 2.141 & 303 & 1,142 & 1,123 & 1,160 \\
\hline & 0 Meningitis (otras en 3) & 33 & 38 & -5 & 0,868 & 0,767 & 0,983 \\
\hline & 1 Enfermedad de Alzheimer & 1.237 & 962 & 275 & 1,286 & 1,248 & 1,325 \\
\hline & $\begin{array}{l}2 \text { Otras enfermedades del sistema nervioso y de los } \\
\text { órganos de los sentidos }\end{array}$ & 1.174 & 1.141 & 33 & 1,029 & 1,010 & 1,048 \\
\hline IX. & Enfermedades del sistema circulatorio $^{3}$ & 31.810 & 32.255 & -445 & 0,986 & 0,984 & 0,988 \\
\hline & 3 Enfermedades cardíacas reumáticas crónicas & 429 & 420 & 9 & 1,021 & 0,996 & 1,047 \\
\hline & 4 Enfermedades hipertensivas & 1.238 & 1.314 & -76 & 0,942 & 0,922 & 0,963 \\
\hline & 5 Infarto agudo de miocardio & 5.803 & 5.837 & -34 & 0,994 & 0,991 & 0,997 \\
\hline & 6 Otras enfermedades isquémicas del corazón & 3.803 & 3.739 & 64 & 1,017 & 1,009 & 1,025 \\
\hline & 7 Insuficiencia cardiaca & 4.694 & 5.164 & -470 & 0,909 & 0,899 & 0,919 \\
\hline & 8 Otras enfermedades del corazón & 3.968 & 3.920 & 48 & 1,012 & 1,003 & 1,022 \\
\hline & 9 Enfermedades cerebrovasculares & 9.540 & 9.531 & 9 & 1,001 & 0,998 & 1,004 \\
\hline & 0 Aterosclerosis & 1.268 & 1.184 & 84 & 1,071 & 1,051 & 1,091 \\
\hline & 1 Otras enfermedades de los vasos sanguíneos & 1.067 & 1.146 & -79 & 0,931 & 0,911 & 0,951 \\
\hline $\mathrm{X}$. & Enfermedades del sistema respiratorio & 10.874 & 10.877 & -3 & 1,000 & 0,995 & 1,004 \\
\hline & 2 Influenza (gripe) & 344 & 337 & 7 & 1,021 & 1,003 & 1,039 \\
\hline & 3 Neumonía & 1.766 & 2.019 & -253 & 0,875 & 0,859 & 0,891 \\
\hline & $\begin{array}{l}4 \text { Enfermedades crónicas de las vías respiratorias inferiores } \\
\text { (excepto asma) }\end{array}$ & 4.563 & 4.574 & -11 & 0,998 & 0,993 & 1,003 \\
\hline 65 & 5 Asma & 253 & 264 & -11 & 0,958 & 0,926 & 0,992 \\
\hline & 6 Insuficiencia respiratoria ${ }^{4}$ & 880 & 505 & 375 & 1,743 & 1,642 & 1,849 \\
\hline & 7 Otras enfermedades del sistema respiratorio & 3.068 & 3.178 & -110 & 0,965 & 0,954 & 0,977 \\
\hline XI. & Enfermedades del sistema digestivo & 4.750 & 4.734 & 16 & 1,003 & 0,999 & 1,008 \\
\hline & 8 Úlcera de estómago, duodeno y yeyuno & 256 & 258 & -2 & 0,992 & 0,964 & 1,021 \\
\hline & 9 Enteritis y colitis no infecciosas & 64 & 61 & 3 & 1,049 & 0,978 & 1,125 \\
\hline & 0 Enfermedad vascular intestinal & 635 & 630 & 5 & 1,008 & 0,996 & 1,020 \\
\hline & 1 Cirrosis y otras enfermedades crónicas del hígado & 1.769 & 1.696 & 73 & 1,043 & 1,031 & 1,056 \\
\hline & 2 Otras enfermedades del sistema digestivo & 2.026 & 2.089 & -63 & 0,970 & 0,959 & 0,981 \\
\hline XII. & Enfermedades de la piel y del tejido subcutáneo & 201 & 185 & 16 & 1,086 & 1,032 & 1,144 \\
\hline XIII. & $\begin{array}{l}\text { Enfermedades del sistema osteomuscular y del tejido } \\
\text { conjuntivo }\end{array}$ & 682 & 665 & 17 & 1,026 & 1,007 & 1,045 \\
\hline & 4 Artritis reumatoide y osteoartrosis & 99 & 96 & 3 & 1,031 & 0,978 & 1,088 \\
\hline & 5 Osteoporosis y fractura patológica & 371 & 373 & -2 & 0,995 & 0,973 & 1,017 \\
\hline & $\begin{array}{l}6 \text { Otras enfermedades del sistema osteomuscular y del } \\
\text { tejido conjuntivo }\end{array}$ & 212 & 195 & 17 & 1,087 & 1,032 & 1,145 \\
\hline XIV. & Enfermedades del sistema genitourinario & 1.721 & 1.715 & 6 & 1,003 & 0,992 & 1,015 \\
\hline & 7 Enfermedades del riñón y del uréter & 1.353 & 1.348 & 5 & 1,004 & 0,990 & 1,017 \\
\hline 78 & 8 Enfermedades de los órganos genitales masculinos & 47 & 46 & 1 & 1,022 & 0,930 & 1,123 \\
\hline & $\begin{array}{l}9 \text { Enfermedades de los órganos genitales femeninos y } \\
\text { trastornos de la mama }\end{array}$ & 15 & 11 & 4 & 1,364 & 0,938 & 1,982 \\
\hline
\end{tabular}


Tabla 4 (Cont.)

Lista INE de 102 grupos de causas. Diferencias, razones de comparabilidad e intervalos de confianza al 95\% entre CIE-10 y CIE-9. Proyecto COMPARA - España 1999

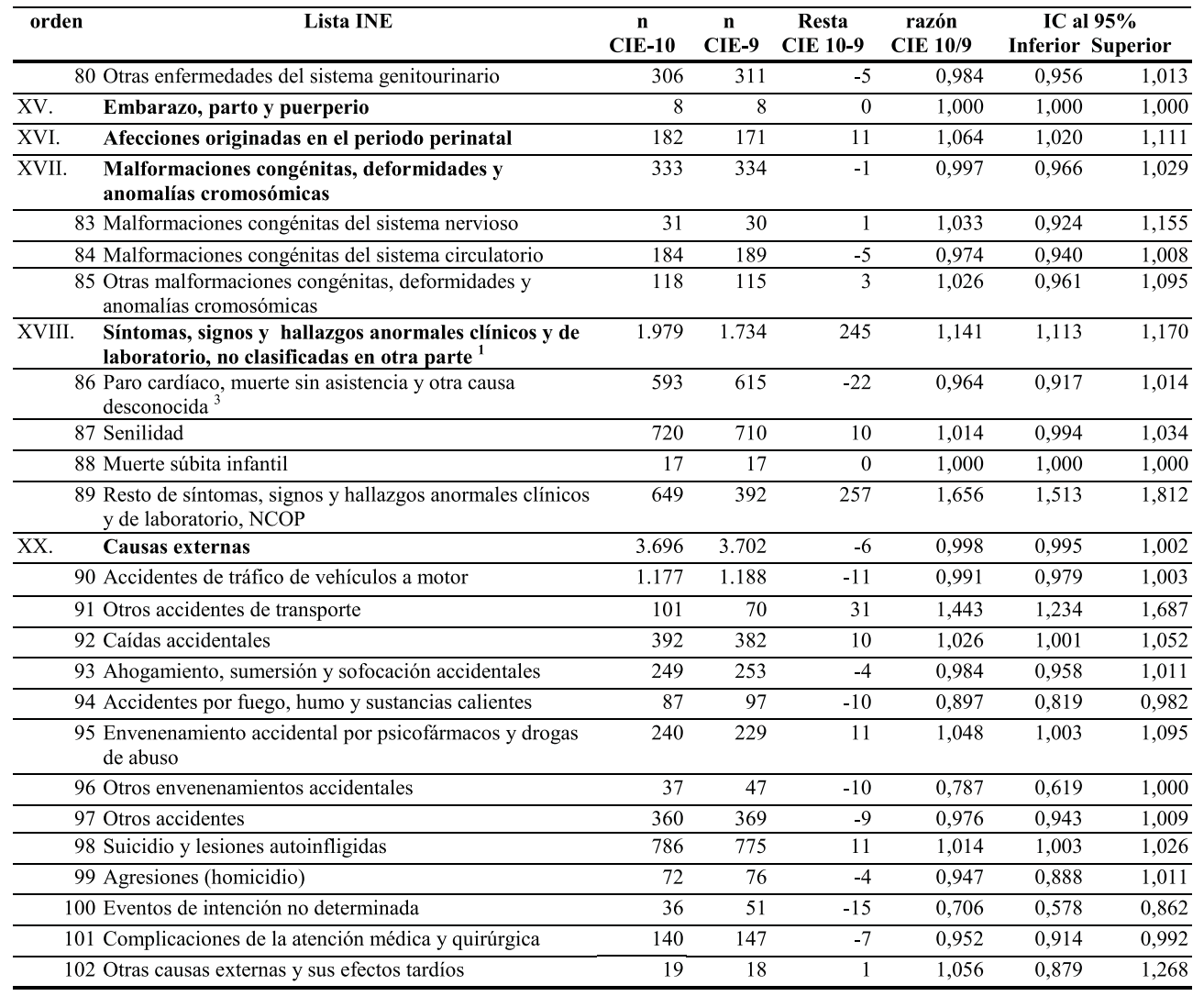

CIE $=$ Clasificación Internacional de Enfermedades

Orden se refiere a grandes causas en los números romanos y a grupos de causas en los árabes.

1 Por motivos de comparabilidad, se incluye VIH+ (R75) (795.8) en el cap. I. Enf. infecciosas y parasitarias, aunque la CIE-10 lo encuadra en el cap. XVIII Síntomas, signos y estados morbosos mal definidos.

2 Por motivos de comparabilidad, se desagrega el S. mielodisplásico (D46) (289.8) ya que la CIE-9 lo encuadraba en el capítulo IV. Enfermedades de la sangre y de los órganos hematopoyéticos.

3 Por motivos de comparabilidad, se excluye el Paro cardíaco (R98,R99) (427.5) de las enf. del sist. circulatorio, ya que la CIE-10 lo encuadra en el capítulo XVIII. Síntomas, signos y estados morbosos mal definidos.

4 Por motivos de comparabilidad con la CIE-10, se desagrega la Insuficiencia respiratoria (J96) (786.0) ya que la CIE-9 lo encuadraba en el capítulo XVIII. Síntomas, signos y estados morbosos mal definidos.

$\mathrm{NCOP}=$ no codificadas en otra parte.

Si ambos límites del intervalo de coinfianza (IC al 95\%) son >1 ó <1, la razón de comparabilidad es estadísticamente significativa.

ciones por distintas causas del sistema respiratorio, entre ellas la neumonía. El mismo esquema se reproduce en el aumento de la diabetes mellitus (48 casos y $2,1 \%$ ).
En los trastornos mentales y del comportamiento se produce una reducción del 7,4\% (202 defunciones) por la salida de la enfermedad de Alzheimer al sistema nervioso y 
Tabla 5

Epígrafes de las listas de Murcia distintos de la lista INE. Diferencias, razones de comparabilidad e intervalos de confianza al $95 \%$ entre CIE-10 y CIE-9. Proyecto COMPARA - España 1999

\begin{tabular}{|c|c|c|c|c|c|c|}
\hline Epígrafes propios de las listas de Murcia & $\begin{array}{c}\text { n } \\
\text { CIE-9 }\end{array}$ & $\begin{array}{c}n \\
\text { CIE-10 }\end{array}$ & $\begin{array}{c}\text { resta } \\
\text { CIE 9-10 }\end{array}$ & $\begin{array}{c}\text { razón CIE } \\
10 / 9\end{array}$ & \multicolumn{2}{|c|}{$\begin{array}{c}\text { IC al } 95 \% \\
\text { Inferior Superior }\end{array}$} \\
\hline Cáncer de vesícula biliar y vías extrahepáticas & 315 & 312 & 3 & 1,010 & 0,981 & 1,039 \\
\hline Cáncer del útero & 412 & 424 & -12 & 0,972 & 0,954 & 0,990 \\
\hline Enfermedad de Hodgkin & 55 & 58 & -3 & 0,948 & 0,865 & 1,039 \\
\hline Linfomas no Hodgkin & 580 & 582 & -2 & 0,997 & 0,978 & 1,015 \\
\hline Neoplasias malignas (cáncer) & 21.330 & 21.276 & 54 & 1,003 & 1,001 & 1,004 \\
\hline Resto neoplasias no-malignas & 619 & 643 & -24 & 0,963 & 0,936 & 0,990 \\
\hline Enfermedades isquémicas del corazón & 9.606 & 9.576 & 30 & 1,003 & 1,000 & 1,006 \\
\hline Enfermedades del hígado & 1.913 & 1.891 & 22 & 1,012 & 1,003 & 1,020 \\
\hline
\end{tabular}

CIE = Clasificación Internacional de Enfermedades

Si ambos límites del intervalo de confianza (IC 95\%) son $>1$ o $<1$, la razón de comparabilidad es estadísticamente significativa.

de los órganos de los sentidos. En su reducción también contribuye en menor cuantía, la salida de casos hacia las enfermedades del sistema circulatorio. Asimismo, una mayoría de muertes por otros trastornos mentales y del comportamiento mejoran su especificidad al trasvasarse a trastornos mentales orgánicos pre/seniles (-131 casos y $-5,2 \%)$. A la vez se incrementa el grupo del sistema nervioso y de los órganos de los sentidos (14,2\% y 303 casos), en su mayoría por la anexión de trastornos mentales, en los que resaltan las demencias de la CIE-9, motivo casi exclusivo del aumento de la enfermedad de Alzheimer (28,6\% y 275 casos).

Las enfermedades del sistema circulatorio sufren un ligero descenso porcentual (-1,4\% y -445 casos), aunque con relevantes movimientos internos originados por la exclusión de la insuficiencia cardiorrespiratoria y por la salida al aparato respiratorio y a enfermedades mentales -esencialmente, las antiguas demencias-.

A la disminución de la insuficiencia cardiaca $(-9,1 \%)$ contribuyen la pérdida de la mencionada insuficiencia cardiorrespiratoria, a la que se suma la insuficiencia respiratoria y otras patologías respiratorias. Mientras que la bajada de las enfermedades hiper- tensivas $(-5,8 \%)$ contribuye la insuficiencia cardiaca (82 casos). El cambio de CIE no produce impacto significativo en la enfermedad cerebrovascular al mantenerse con escasas variaciones, aunque pierde casos hacia los trastornos orgánicos pre/seniles.

El infarto agudo de miocardio decrece ($0,6 \%)$ por otras enfermedades isquémicas del corazón, con la paradoja del aumento $(0,3 \%)$ de la enfermedad isquémica cardiaca (tabla 5) vía otras enfermedades del corazón y de los vasos sanguíneos y de la insuficiencia cardiaca. Otras enfermedades del corazón $(1,2 \%)$ incluyen casos de paro e insuficiencia cardiaca. A su vez la aterosclerosis se magnifica $(7,1 \%)$ al expandirse con casos de otras enfermedades de los vasos sanguíneos $(-6,9 \%)$.

Las enfermedades respiratorias no presentan cambio neto, al contrarrestar los casos recibidos con los cedidos a las enfermedades cardiovasculares entre otros grupos (tabla 3 ). En la magnificación de la insuficiencia respiratoria $(74,3 \%$ ) (tabla 4) intervienen otras enfermedades del sistema respiratorio, la insuficiencia cardiaca, la neumonía, otras enfermedades del corazón y varias entidades en escasa cuantía. Mientras que el descenso de la neumonía $(-12,5 \%)$ radica en la secesión 
de la insuficiencia respiratoria, de los trastornos mentales orgánicos pre/senil, de la diabetes mellitus y de las enfermedades cerebrovasculares, entre otros numerosos epígrafes.

Se mantiene estable el grupo de las enfermedades digestivas, mientras que la cirrosis y otras enfermedades crónicas del hígado aumentan $(4,3 \%)$ por la inclusión de otras enfermedades digestivas y de las hepatitis víricas. Características que se repiten en menor proporción para el conjunto de las enfermedades del hígado $(1,2 \%)$ (tabla 3 ). Las enfermedades de la piel y del tejido subcutáneo y las afecciones perinatales experimentan un aumento del 8,6\% y del 6,4\%, respectivamente, generado por un escaso diferencial.

Las enfermedades osteomusculares y del tejido conjuntivo crecen un 2,6\% (17 casos), sin menciones de interés, a pesar de incluir a la poliarteritis nudosa y la gota.

Se mantienen las enfermedades del sistema genitourinario, las causas ligadas a la maternidad y las malformaciones congénitas, sin patologías individuales reseñables.

El capítulo de «síntomas, signos y hallazgos anormales clínicos y de laboratorio, no clasificados en otra parte» (entidades mal definidas) sube un $14,1 \%$, debido a la inclusión (390 casos) de enfermedades del aparato circulatorio, en su mayoría insuficiencias cardiorrespiratorias (351 casos).

Las causas externas permanecen estables, al igual que su componente, los accidentes de tráfico. Contradictoriamente se magnifica la proporción de otros accidentes de transporte $(44,3 \%)$ originada por la cesión de accidentes de tráfico de vehículos a motor desde la CIE-9.

En el conjunto de las 88.048 defunciones obtenemos en la lista INE, un índice kappa de $97,0 \%$ para los 17 grandes grupos de causas y del 95,4\% para los 102 grupos de cau- sas, siendo del $95,6 \%$ para la lista MU-86 y 95,9\% para MU-66.

\section{DISCUSIÓN}

El impacto del cambio de CIE en España globalmente considerado por los índices kappa, es de menor cuantía. El impacto es consecuencia directa de la elaboración de las correspondencias de códigos en las listas de causas. La exactitud resultante se debe a que agrupamos con causas más específicas en base a la experiencia del grupo COMPA$\mathrm{RA}^{6}$; al obtener un $3 \%$ de defunciones mal clasificadas en los grandes grupos de causas a cuatro caracteres, frente al $5,1 \%$ por grandes grupos de causas ${ }^{6}$. Estudio que abarca casi la cuarta parte de las defunciones de 1999 en España, presentó las principales diferencias empíricas con la CIE-9, así como las ventajas e inconvenientes del diseño y ejecución del proyecto.

No observamos patrones individuales de concordancia diferenciales en las listas tabulares entre los centros participantes (rango de índices kappa de 93,6\% a 96,9\%. La formación sobre las normas de codificación en mortalidad de la CIE-10 se realizó en un curso único para todo el personal codificador con participación docente nacional e internacional ${ }^{22}$. Previamente, los centros codificadores coordinados por el INE habían actualizado a la CIE-10 el manual de causas de defunción en sus acuerdos sobre casos particulares, causas según la edad, términos que no figuran en la CIE, siglas, y códigos infrecuentes o erróneos, entre otros ${ }^{23}$.

La variante de Murcia aporta más recursos a la equiparación de causas de muerte en España y la posibilidad de contrastar internacionalmente los resultados (más información en www.MurciaSalud.es/compara). En determinados epígrafes las propuestas más estrictas no han sido eficientes, como en los accidentes de tráfico, en los que se postula, incluso un criterio más amplio, como de 
accidentes de trasporte terrestre (códigos V01-V89 o V99) ${ }^{24,25}$. A pesar de la ampliación de rúbricas, en las listas presentadas, no se desglosan las tuberculosis respiratorias del conjunto.

El índice kappa verifica como dos mediciones del mismo tipo concuerdan - p. ej. dos observadores midiendo la presión sanguínea del mismo paciente-. Se puede usar para calcular la falta de concordancia de la causa básica de defunción en CIE-10 con la propia de la CIE-9. Sin embargo, no es este el tema abordado, ya que sabemos que las dos clasificaciones son diferentes, y lo que queremos medir es el tamaño del cambio al movernos de una a otra revisión, para poder interpretar las tendencias temporales en las estadísticas rutinarias de causas de muerte.

Cuando las razones de comparabilidad son iguales a uno, se considera que no hay cambios entre CIE, aun sabiendo que el mismo número de muertes atribuido en las dos revisiones a una determinada causa no indica necesariamente que no esté afectado por la introducción de la nueva revisión, dada la posibilidad de que los cambios múltiples se compensen unos con otros, como se puede observar en los resultados de este trabajo. Por otro lado, las razones menores o mayores de uno indican que no existe una total equivalencia entre las dos revisiones. Un número menor de defunciones en un grupo de causas puede ser debido a que alguna causa del grupo haya pasado a otro en la CIE10 , y un número mayor de muertes asignadas a un grupo de causas en CIE-10 que en CIE-9, puede ser debido a que no exista una total equivalencia entre revisiones. De una forma práctica, las razones de comparabilidad se pueden utilizar para establecer las correspondencias entre la CIE-9 y 10 en España, cuando las listas tabulares sean las mismas que las aquí presentadas.

Al estratificar por sexo se reducen los efectivos por causa, y en determinados casos su frecuencia es muy baja. En general, las razones de comparabilidad según sexo mantienen el signo y magnitud en el intervalo de confianza de la razón total de comparabilidad.

El análisis realizado es homólogo al aplicado por el Office for National Statistics para Inglaterra-Gales, el implementado en Escocia por el General Register Office for Scotland, y el del National Vital Statistics System - Centers for Disease Control and Prevention de EEUU y la Health Statistics Division of Statistics de Canadá. Las disimilitudes en la presentación de resultados radican en que los informes escocés y francés no incluyen los intervalos de confianza de las razones de comparabilidad, y que la versión final con datos de Inglaterra - País de Gales desglosa las razones según el sexo ${ }^{26}$. Tanto en Francia, como Gran Bretaña, EEUU o Canadá la imputación de la causa básica es automática en el $80-85 \%$ de los certificados (que excluyen muertes judiciales y perinatales) con 1.852.671 defunciones en EEUU de 1996, 551.093 en Inglaterra-Gales, 60.281 en Escocia $^{27}$, 98.847 en Canadá28 y 53.869 (el 10\% de las defunciones anuales) en Francia $^{29}$, siendo el resto de países del año 1999.

Observamos que al añadir entidades singulares a las correspondencias por grandes causas de muerte varía el signo de la relación entre revisiones al comparar con los resultados obtenidos exclusivamente entre grandes causas $^{6}$. Así sucede por las correspondencias VIH y SIDA entre las enfermedades infeccioso-parasitarias y las endocrino-nutricionales-metabólicas; y en las enfermedades respiratorias por el reparto de la insuficiencia cardiorrespiratoria entre éstas y las entidades mal-definidas. En EEUU ${ }^{20}$, Reino Unido $^{21,27}$ y Canadá ${ }^{28}$ no incluyen códigos singulares en las correspondencias en listas tabulares por grandes causas de muerte, con la excepción de la OPS.

El SIDA presenta una razón de comparabilidad positiva y significativa al igual que en EEUU, Canadá y en Inglaterra-Gales en 
hombres. Las hepatitis víricas descienden también en EEUU, Canadá y España en porcentaje similar $(12,3 \%, 12,7 \%$ y $16,5 \%)$, sin que varíen en Inglaterra-Gales.

El mínimo aumento de las neoplasias malignas está muy próximo al de EEUU, Canadá y Gran Bretaña ${ }^{21}(0,3 \%, 0,7 \%, 0,3 \%$ y $1,2 \%$, respectivamente). Por el contrario el efecto de la CIE-10 hace descender levemente al cáncer de pulmón en EEUU, España y Reino Unido. Mientras que el síndrome mielodisplásico se incrementa en España, sin que se individualice en las listas anglosajonas.

Con el cambio de revisión la diabetes mellitus aumenta un poco en España $(2,1 \%)$, menos en Estados Unidos $(0,8 \%)$ y algo más en Reino Unido y Canadá (4,0\%).

En la novena revisión cuando se menciona la enfermedad de Alzheimer sin demencia, se incluye en la sección del sistema nervioso, mientras que en la décima revisión toda mención de Alzheimer (con o sin demencia) se incluye en el sistema nervioso. Así, la enfermedad de Alzheimer aumenta mucho en España, EEUU, Inglaterra-Gales y Canadá, por la transferencia desde las demencias preseniles en CIE-9 y por un criterio laxo de imputación ante cualquier mención de Alzheimer en CIE-10 (regla 3), además de los problemas de validez del diagnóstico de las demencias en general, que suelen exigir complejas pruebas complementarias de exclusión de otras entidades. Esta enfermedad no se contempla en la lista corta de Eurostat. Una solución, sería la unión de la demencia y el Alzheimer, tal y como proponen Becker y colaboradores en el ordenamiento de las principales causas de muerte ${ }^{30}$.

La enfermedad isquémica cardiaca aumenta ligeramente en España y Francia (ambas 0,3\%), algo más en Inglaterra-Gales $(0,5 \%$ en hombres y $0,7 \%$ en mujeres), se mantiene en Canadá, y disminuye en EEUU.
Sin embargo el infarto agudo miocardio (IAM) disminuye en España, InglaterraGales, Estados Unidos y Canadá. En nuestro país se ha comprobado que la mortalidad por enfermedad isquémica cardiaca es una rúbrica sensible en la detección de IAM, pero que suele estar infranotificado ${ }^{17}$. La enfermedad cerebrovascular no varía en España, pero aumenta en EEUU (5,9\%), Inglaterra-Gales (8,6\%-12,5\%), Escocia (6,0\%) y Canadá $(6,9 \%)$. Los estadounidenses lo atribuyen a la regla 3 y a transferencia de las antiguas neumonías en CIE-9. Mientras que la insuficiencia cardiaca disminuye $(-9,1 \%)$ en España, aumenta en EEUU $(4,1 \%)$ y Canadá $(3,5 \%)$, las diferencias se pueden deber, a una certificación más precisa en Norteamérica $^{31}$.

La neumonía baja (-12,5\%) en España, y drásticamente en EEUU (-30,4\%), Inglaterra-Gales $(-44,3 \%$ a $-35,1 \%)$, Escocia ($33,6 \%)$ y Canadá $(-46,8 \%)$ por la aplicación de la CIE-10. La gripe se incrementa escasamente (7 casos) en España, sin afectarse en EEUU o en Gran Bretaña. Estas diferencias reflejan parte del cambio de interpretación de la selección de la causa básica de defunción en las neumonías y bronconeumonías por la regla 3 -originalmente aceptadas como complicación de cualquier enfermedad-, aunque deben haber más factores, porque esa era la postura implantada en España en 1989. La OMS por indicación del Update Reference Committee $(U R C)$ ha restringido su aplicación en la regla 3 desde el año $2003^{28}$.

Las cirrosis y otras enfermedades crónicas del hígado suben por efecto de la CIE10, en España (4,3\%), EEUU (3,7\%), Inglaterra-Gales $(5,0 \%-9,4 \%)$ o Canadá $(6,3 \%)$.

En anomalías congénitas y afecciones perinatales sería deseable valorar el impacto del cambio sobre listas de mortalidad infantil o perinatal como han hecho otros países ${ }^{32}$, pero dado el número de defunciones infantiles (401 casos) a cruzar con los epígrafes de las listas INE (54 en mortalidad infantil y 93 
en perinatal) ${ }^{33}$, desaconsejan por diseño su comparación.

El aumento de las entidades mal definidas está generado por el paro cardíaco y la insuficiencia cardiorrespiratoria. Las insuficiencias respiratoria, cardiorrespiratoria y otras causas pasaron a ser motivo de mejora de la información en el año 2002 en España, tras una recomendación del $U R C^{34}$, lo que ocasionará una cierta inadecuación de las razones de comparabilidad, por disminución del monto del resto de las entidades mal definidas y de todo el grupo, pero difícilmente anulará su incremento.

En relación a las causas externas no apreciamos cambios relevantes, siendo difícil de valorar por categorías. En Francia informan de su descenso (4,6\% y 1.980 muertes) debido a importantes modificaciones en este capítulo y en la interpretación de los certificados de defunción, especificando que las fracturas de causa no especificada pasan a accidente SAI (sin más indicación, en latín) (X59) desde un código propio del grupo de caídas. Mientras que en Canadá no hay variación significativa en las causas externas. Sin embargo en EEUU indican un aumento del 3,1\% en accidentes (unintentional injuries, códigos V01-X59, Y85-Y86) desde las defunciones naturales (por ejemplo, neumonía y paro cardíaco) por cambios en las reglas 3 y A (reselección de la causa básica cuando es senilidad $\mathrm{u}$ otras entidades mal definidas). En EEUU y Canadá los accidentes incluyen el decremento de los accidentes de vehículos a motor $(-14,7 \%$ y $-1,9 \%$, respectivamente), motivado porque ahora la palabra motor se debe explicitar y antes bastaba su ocurrencia sobre la calzada; también obtienen un descenso de las caídas $(-15,9 \%$ y $-49,8 \%$, respectivamente) que razonan en el mismo sentido que los franceses, aunque el resultado esté más próximo a Inglaterra-Gales $(-44,4 \%)$ que a Escocia ( $\sin$ cambios). El informe de Inglaterra-Gales resalta, que las correspondencias en las causas externas no se afectan, que no se encuen- tra el equivalente a los accidentes de tráfico de vehículos a motor, que la información sobre drogas ha perdido la individualización que tenía en CIE- $9^{24}$, y que las caídas disminuyen aún más $(-25,6 \%$ a $-56,3 \%)$ que en otros informes ${ }^{35}$. Por esa razón y según la OMS, a partir de enero del 2006, la categoría X59 tendrá como cuartos caracteres: ' $\underline{O}$ Exposición a factores no especificados causando fractura' y '9 - Exposición a factores no especificados causando otras lesiones y las no especificadas'. De esa forma se pueden rescatar las fracturas no especificadas y considerarlas o no caídas, de acuerdo a la edad y otros factores, en el momento del análisis y tabulación. Debido a la diferente organización de la CIE-10, muchos de los accidentes de tránsito de vehículo a motor, se están codificando en categorías residuales, dando la apariencia de que son otros accidentes de transporte. Los británicos resaltan que se gana más información con CIE-10 en el envenenamiento por drogas si se codifica la naturaleza de la lesión / causa secundaria, aunque se realice en pocos países ${ }^{36}$.

La consideración sobre la actualización de las entidades mal definidas es análoga a toda la terminología médica que modifique su interpretación en el transcurso de la CIE10 , que afecte a las causas de las listas por su potencial repercusión en las razones de comparabilidad. En este sentido y en términos generales, los británicos comentan que existe un periodo de adaptación ${ }^{21}$, situación que pudo reproducirse en España en el año de implantación de la CIE-10.

Otra cuestión es si las razones de comparabilidad se alteran por grupos específicos de edad o al ajustar por edad. Estos temas se han tratado en los informes del Office for National Statistics británico sin que encuentren diferencias apreciables entre las razones ajustadas por edad, o específicas de grupos de edad ${ }^{21}$, en una población con más efectivos y más envejecida que la española. En la función de mortalidad las edades más ancianas, representan un peso mayor y principal. 
En nuestro caso el $82 \%$ de las defunciones ocurre en personas mayores de 64 años. No obstante si se quieren aplicar para describir tendencias en grupos específicos de edad más jóvenes, las razones podrían variar. Sin que las circunstancias anteriores, se observen, en su inmensa mayoría, diferencias estadísticamente significativas, con más defunciones, como en Inglaterra y Gales ${ }^{21}$. Las causas sexo-específicas tabuladas en nuestas listas introducen un necesario contrapunto al pertinente análisis según sexo ${ }^{37}$.

La OMS dispone de una red de Centros Colaboradores de las Clasificaciones Internacionales para el uso, desarrollo y difusión en las estadísticas y los sistemas de información de salud ${ }^{38,39}$. Al igual que Australia, Reino Unido o Estados Unidos son centros colaboradores en su idioma; España por la relevancia de su Sistema Nacional de Salud, podría formar parte de los mismos, produciendo sinergias, con el centro oficial para el español de Venezuela y con el centro candidato de Méjico, en pos de una mayor proyección internacional.

Para la estadística de la Unión Europea sería un avance obtener razones de comparabilidad para las causas de muerte para la lista de Eurostat, inclusive con la información de los estudios de correspondencias realizados en los distintos estados miembros de la Unión Europea; si se establece la viabilidad en su diseño y ejecución.

En España deberían tenerse en cuenta las razones de comparabilidad en la valoración de la consecución de objetivos de mortalidad de los Planes de Salud ${ }^{20}$. A la vez que los temas pendientes de la estadística de mortalidad, se deberían encarar desde la coordinación institucional, más que del voluntarismo profesional.

\section{AGRADECIMIENTOS}

A Jerónimo Martínez, Consuelo Martínez y Diego Salmerón por sus aportaciones al manuscrito. A las personas e instituciones del proyecto COMPARA y al Área de Estadísticas Sanitarias del Instituto Nacional de Estadística de España. A Clare Griffiths y Cleo Rooney del Office for National Statistics - United Kingdom por sus aclaraciones acerca del uso de las razones de comparabilidad.

El presente trabajo está financiado por el Instituto de Salud Carlos III (Red de Centros C03/09) del Ministerio de Sanidad y Consumo del Gobierno de España.

\section{ANEXO 1}

El grupo COMPARA está formado por las siguientes personas e instituciones públicas locales o autonómicas: María Méndez Méndez, Carmen Murillo Cordobés, Isabel Rojas Vázquez, Miguel Ruiz Ramos de la Consejería de Salud de Andalucía; Carme Borrell Thió, Dolores Martos Hinojosa, Glòria Pérez Albarracín de la Agencia de Salud Pública de Barcelona; Luz Gómez Lastra, Isabel Torcida Boo, Raymundo de la Consejería de Sanidad, Consumo y Servicios Sociales de Cantabria; Lluís Cirera Suárez, Josefina Contreras Gil, José García Rodríguez, Emilia Madrigal de Torres, Consuelo Martínez López, Carmen Navarro Sánchez, Miguel Rodríguez Barranco y Aurelio Tobías Garcés de la Consejería de Sanidad de Murcia; Concha Moreno Iribas y Yugo Floristán Floristán del Instituto de Salud Pública de Navarra; Covadonga Audicana Uriarte, Tomás Lizárraga Sánchez y Josefina Rodríguez Beascoechea del Departamento de Sanidad del Gobierno Vasco.

\section{BIBLIOGRAFÍA}

1. Vázquez E. Taller de codificación con la CIE-10 revisión. SEENOTA, 1996 mayo-agosto. p. 5.

2. Cirera L, Pérez G, Ramos M, Audicana C, Fernández A, Vázquez E. Aspectos clave de la nueva revisión de la Clasificación Internacional de Enfermedades: la CIE-10. SEENOTA 1996; 4-5. 
3. Cirera L. CIE-10 [recensión bibliográfica]. Med Clin 1997;109:531.

4. Audicana C, Cirera L, Becker R. Caracterización de la $10^{\text {a }}$ Revisión de la Clasificación Internacional de Enfermedades (CIE-10). Principales diferencias con la novena revisión en su aplicación en mortalidad. En: Cirera L, Vázquez E, eds. La implantación en España de la Clasificación Internacional de Enfermedades $10^{\mathrm{a}}$ Revisión [CIE-10]. Santiago de Compostela: Sociedad Española de Epidemiología, 1998; p. 55-69.

5. Diferencias entre la CIE-9 y CIE-10. Anónimo. En: Instituto Nacional de Estadística. Manual de causas de defunción (CIE-10). Versión 4.0. Madrid: INE; 2005. Documentos de trabajo. p. 1-4.

6. Ruiz M, Cirera L, Pérez G, Borrell C, Audicana C, Moreno $\mathrm{C}$ et al. Comparabilidad entre la novena y la décima revisión de la Clasificación Internacional de Enfermedades aplicada a la codificación de la causa de muerte en España. Gac Sanit 2002; 16(6): 526-32.

7. Instituto Nacional de Estadística. Anexo 3. Listas reducidas de causas de defunción. En: INE. Manual de causas de defunción (CIE-10). Versión 4.0. Madrid: INE; 2005. Documentos de trabajo. p. 110. www.ine.es/daco/daco42/sanitarias/listas_ 02.doc

8. Área de Análisis Epidemiológico y Situación de Salud. Lista de causas seleccionadas. Centro Nacional de Epidemiología. Instituto de Salud Carlos III. <Consulta Diciembre 2005> http:// 193.146.50.130/htdocs/mortal/mortal2001/wlista_cie10.htm

9. Hatton F, Boileau J; Renaud G, Pavillon G. Bridge coding between ICD-9 and ICD-10 on 5,179 cases. En: Meeting of Heads of WHO Collaborating Centres for the Classification of Diseases, Tokyo, Japan, 15-21 October, 1996. Geneva: World Health Organization; 1996. (Unpublished document $\mathrm{HO} / \mathrm{HST} / \mathrm{ICD} / \mathrm{C} / 96.30)$.

10. Organización Mundial de la Salud. Listas especiales de tabulación para la mortalidad y la morbilidad. En: OPS-OMS. Manual de la Clasificación Estadística Internacional de Enfermedades, Traumatismos y Causas de Defunción. Novena Revisión. Vol. 1. Washington DC: OPS; 1978. p. 1143-1154.

11. Organización Panamericana de la Salud. Manual de la Clasificación Estadística Internacional de Enfermedades, Traumatismos y Causas de Defunción. Novena Revisión. Vols. 1 y 2. Washington DC: OPS; 1978.
12. Organización Panamericana de la Salud. Clasificación Estadística Internacional de Enfermedades y Problemas Relacionados con la Salud. Décima revisión. (CIE-10) Manual de instrucciones. Vol. 1. Washington DC: OPS; 1995.

13. Instituto Nacional de Estadística. Descripción de errores y avisos en variables sanitarias. Estadística de partos (CIE-10). Documento técnico (versión 2.0). Madrid: INE; 2001. (Documento mimeografiado).

14. Instituto Nacional de Estadística. Manual de causas de defunción (CIE-10). Versión 4.0. Madrid: INE; 2005. Documentos de trabajo.

15. Instituto Nacional de Estadística. Manual de causas perinatales de defunción (CIE-10). Documento Técnico (Versión 1.1). Madrid: INE; 2000.

16. Organización Panamericana de Salud. Nueva lista OPS 6/67 para la tabulación de datos de mortalidad CIE-10. Bol Epidemiol 1999; 20(3): 4-9 [marzo de 2005] http://www.paho.org/spanish/sha/BE_ v20n3.pdf

17. García J, Cirera L, Tormo MJ, Martínez C, Contreras J, Navarro C. Utilidad del boletín estadístico de defunción para la identificación de muertes extrahospitalarias en un registro poblacional de infartos agudos de miocardio. Rev Esp Cardiol 2001; 54: 1041-1047.

18. Cirera L, Navarro C. Validez de la certificación de la muerte por cáncer en la Comunidad de Murcia. Oncología 2002; 25(5): 38-46.

19. Fleiss JL. Statistical Methods for Rates and Proportions. NY: John Wiley \& Sons; 1981. p. 218-225.

20. Anderson RN, Arialdi MM, Hoyert DL, Rosenberg HM. Comparability of Cause of Death between ICD-9 and ICD-10: Preliminary Estimates. Hyattesville, Maryland, USA: National Center for Health Statistics. National Vital Statistics reports 2001; 49 (2): 1-32.

21. Rooney C, Grifftihs C, Cook L. The implementation of ICD-10 for cause of death coding - some preliminary results from the bridge coding study. Health Statistics Quarterly 2002; 13: 31-41.

22. Instituto Nacional de Estadística. Curso sobre el uso de la Décima Revisión de la Clasificación Internacional de Enfermedades de la OMS. Madrid: INE; 1998.

23. Instituto Nacional de Estadística. Manual de causas de defunción (CIE-10). Versión 1.0. Madrid: INE; 1998. Documentos de trabajo. 
24. Griffiths C, Rooney C. Results of the England and Wales ICD-10 comparability study: the effect on main injury and external causes. Meeting of WHO collaborating Centres for the Family of International Classifications. Cologne, Germany 19-25 October 2003. WHO/HFS/CAS/C/03.84 [Consulta marzo 2005] www.rivm.nl/who-fic/Colognepapers/ cologne84.rtf

25. Motor vehicle traffic accidents in Mexico, Changes resulting from implementation of ICD-10, Mexican Centre for the Classification of Diseases, WHO/GPE/CAS/C/01.86 www.who.int/classifications/network/en/report2001.pdf

26. Office for National Statistics. United Kingdom. Comparability Ratios for major causes of death by sex. [Consulta marzo de 2005] www.statistics. gov.uk/about/classifications/icd10/downloads/all_ age_comparability_ratios.xls

27. General Register Office for Scotland. Vital Events. Bridge-coding analysis of death in 1999; ICD9 chapter by ICD10 chapter. [Consulta marzo de 2005] www.gro-scotland.gov.uk/files/00tab24.pdf

28. Geran L, Tully P, Wood P, Thomas B. Comparability of ICD-10 and ICD-9 for Mortality Statistics in Canada. Ottawa, Ontario: Minister of Industry. Statistics Canada. Health Statistics Division; 2005.

29. Pavillon G, Boileau J, Renaud G, Lefèvre H, Jougal E. Bridge coding studies ICD9-ICD10 and effects on French mortality dada. En: WHO Family of International Classifications Network Meeting. Reykjavik, Iceland, 24-30 October, 2004. WHOFIC/04.080. [Consulta marzo 2005] http://www. nordclass.uu.se/WHOFIC/reykjavik.htm\#Report

30. Becker R, Silvi J, Ma Fat D, L'Hours A, Laurenti R. A method for Deriving Leading Causes of Death Bull World Health Organ 2006; 84: 297-304.

31. Ganguli M, Rodriguez EG. Reporting of dementia on death certificates: a community study. J Am Geriatr Soc 1999; 47(7):842-9.
32. Dattani N, Rowan S, Rooney C. Updating the ONS (OPCS) hierarchical classification of causes of stillbirth and infant deaths for ICD-10. En: Meeting of Heads of WHO Collaborating Centres for the Classification of Diseases, Brisbane, Australia, 1419th October 2002. World Health Organization; 1996; p.1-5. [Consulta marzo 2005] www.aihw. gov.au/international/who_hoc/hoc_02_papers/bris bane70.doc

33. Instituto Nacional de Estadística. Equivalencia de la lista reducida de mortalidad infantil y perinatal CIE-10 con CIE-9. [Consulta marzo de 2005] www.ine.es/daco/daco42/sanitarias/listas_02.doc

34. Update Reference Committee. Updates ICD-10. URC - WHO. October 2003. p. 34 [Consulta marzo 2005] www3.fhs.usyd.edu.au/ncch/downloads/ official_WHO_updates_combined\%201996\%20to $\% 202003$.pdf

35. Griffiths $\mathrm{C}$ and Rooney C. Introduction of ICD-10 on trends in mortality from injury and poisoning in England and Wales. Health Statistics Quarterly 19, 10-21. [Consulta marzo 2005] www.statistics. gov.uk/downloads/theme_health/HSQ19.pdf

36. Rooney C, Grifftihs C, Cook L. Results of ICD-10 bridge coding Study England and Wales. En: Meeting of Heads of WHO Collaborating Centres for the Classification of Diseases, Brisbane, Australia, 1419 October, 2002. Geneva: World Health Organization; 2002. Document WHO/GPE/CAS/ C/02.71.

37. National Statistics. Comparability ratios for selected causes of death by broad age band and sex, England and Wales, 1999. [Consulta marzo 2005] www.statistics.gov.uk/about/classifications/icd10/ comparability_ratios.asp

38. Collaborating Centres for the WHO Family of International Classifications [Consulta agosto de 2005] www.who.int/classifications/network/collaborating/en/index.html

39. WHO Family of International Classifications Network. [Consulta agosto de 2005] www.who. int/classifications/network/en/Networkpaper.pdf 